\title{
Enemy Within: Long-term Motivation Effects of Deep Player Behavior Models for Dynamic Difficulty Adjustment
}

\author{
Johannes Pfau \\ Digital Media Lab, TZI, \\ University of Bremen \\ Bremen, Germany \\ jpfau@tzi.de
}

\author{
Jan David Smeddinck \\ Open Lab, School of Comp., \\ Newcastle University \\ Newcastle upon Tyne, UK \\ jan.smeddinck@newcastle.ac.uk
}

\author{
Rainer Malaka \\ Digital Media Lab, TZI, \\ University of Bremen \\ Bremen, Germany \\ malaka@tzi.de
}

\begin{abstract}
Balancing games and producing content that remains interesting and challenging is a major cost factor in the design and maintenance of games. Dynamic difficulty adjustment (DDA) can successfully tune challenge levels to player abilities, but when implemented with classic heuristic parameter tuning (HPT) often turns out to be very noticeable, e.g. as "rubberbanding". Deep learning techniques can be employed for deep player behavior modeling (DPBM), enabling more complex adaptivity, but effects over frequent and longer-lasting game engagements, as well as comparisons to HPT have not been empirically investigated. We present a situated study of the effects of DDA via DPBM as compared to HPT on intrinsic motivation, perceived challenge and player motivation in a real-world MMORPG. The results indicate that DPBM can lead to significant improvements in intrinsic motivation and players prefer game experience episodes featuring DPBM over experience episodes with classic difficulty management.
\end{abstract}

\section{CCS Concepts}

-Human-centered computing $\rightarrow$ User models; -Computing methodologies $\rightarrow$ Neural networks; •Applied computing $\rightarrow$ Computer games;

\section{Author Keywords}

Dynamic difficulty adjustment; Player Modeling; Neural Networks; Deep Learning; MMORPGs; Games

\section{INTRODUCTION}

With the ongoing rise of complexity, popularity and content production cost of video game development, consistently balanced challenges that keep players motivated over the long term are becoming hard to attain, especially with large player communities encompassing broad ranges of proficiency. Dynamic Difficulty Adjustment (DDA) [23] denotes the principle of adapting video game challenges to players' abilities - both mental and physical / dexterity - in order to allow motivationfostering flow states [10] to arise. It has been successfully

\footnotetext{
(C) the authors, 2020. This is the author's version of the work. It is posted here for your personal use. Not for redistribution.

The definitive version was published as:

Johannes Pfau, Jan David Smeddinck, and Rainer Malaka. 2020. Enemy Within: Long-term Motivation Effects of Deep Player Behavior Models for Dynamic Difficulty Adjustment. In Proceedings of the $2020 \mathrm{CHI}$ Conference on Human Factors in Computing Systems (CHI '20), 1-10. https://doi.org/10.1145/3313831.3376423
}

deployed in scientific [23, 24, 46] and industrial [7, 16, 55] contexts and is usually accomplished by continuous tuning of core game variables (such as speed, damage or hit ratio). However, these systems are inherently limited to a small number of high-level parameters, require careful tuning of thresholds in heuristic parameter tuning (HPT) [51] and have to be hidden to avoid exploitation; e.g. as not to incentivize players to perform badly on purpose [43]. This results in limited expressivity and complexity of system behavior, as well as in considerable development cost. To address these limitations of classic HPT, we present a novel DDA strategy by implicitly assessing individual player proficiency using Deep Player Behavior Modeling (DPBM) [38] and generating adaptive, personalized challenges. Player behavior, in terms of stateaction decision making, is captured while fighting an in-game opponent and trained onto an individual, initially randomized model. Upon the next encounter, the opponent uses this model generatively by retrieving action probabilities for each game state emerging in an interaction. As a consequence, its decision making approximates the original player's behavior, implicitly representing the particular game proficiency. Evaluating the real-world applicability of DPBM, we aim to answer the following research questions:

- Do players perceive behavior from DPBM as representative of their own decision making?

- Is the players' self-reported intrinsic motivation when interacting with a DPBM opponent higher than for traditional HPT encounters?

- Can we measure a substantial long-term motivation achieved by DPBM?

We hypothesize that an agent that keeps up with the progress of the player, displays similar weaknesses and challenges players to constantly improve or rethink strategies will yield a novel and captivating take on DDA. For the purpose of evaluating the differences between HPT and DPBM, we implemented Eternal Challenge - an adaptive instance dungeon inside of the popular Massively Multiplayer Online Role-Playing Game (MMORPG) Aion [34] - and assessed players' motivation in a field study ( $n=171)$ on an existing private server. After a deployment of four weeks, we were successful in showing a significantly higher long-term usage of the instance compared to all alternatives and that the DPBM opponent contributes significantly more to this motivation than HPT mechanics, 


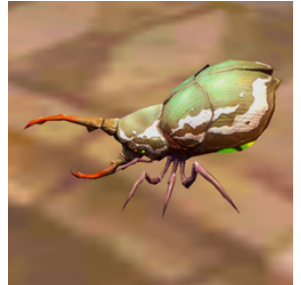

a: frequency

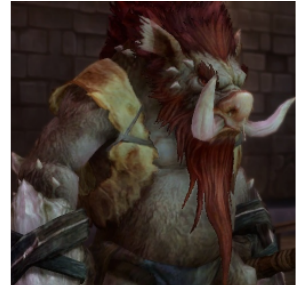

$\beta$ : perseverance

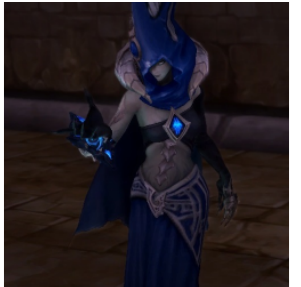

Y: disturbance

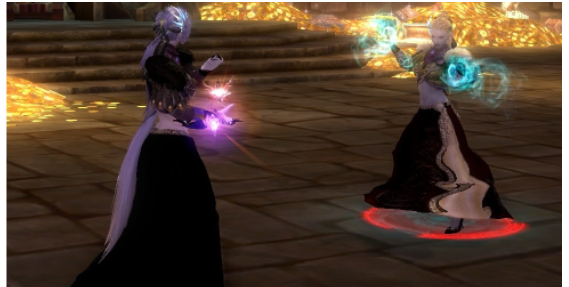

DPBM

Figure 1. Appearance of the different opponents with DDA through HPT variables and DPBM.

based on quantitative - and supported by qualitative - insights. Furthermore, some players stated that they noted the progress of the DPBM opponent in learning their own individual strategies, leading to a unique game experience. We contribute to games user research and inform game development by investigating DPBM as a form of novel DDA in a situated medium- to long-term study, showcasing its distinct potential for fostering intrinsic motivation and demonstrating a working approach with learning adaptive opponents in the wild.

\section{RELATED WORK}

Providing and balancing an accurate level of difficulty is critical for keeping players constantly engaged [2, 23]. Disparities can ultimately lead to boredom/underload or frustration/overload, which make for two of the main causes why players stop playing games [11]. Since individual skill and its progression are hard to foresee throughout potentially large player bases and difficulty and it's progression can not be defined or programmed precisely, the field of DDA attempts to regulate emergent mismatches dynamically. To estimate imbalanced challenge-proficiency-discrepancies, various assessment techniques have been researched, such as success probability estimation $[24,32]$ or biofeedback [22, 25, 35]. However, When it comes to adjusting this difficulty, most approaches focus on HPT (apart from procedural world generation $[26,57,50])$, even in the most recent advancements [3, $4,8,17,18,40]$.

Opponents that imitate the player character exist in numerous commercial games, perhaps most notably the recurring Dark Link in the The Legend of Zelda series [15], the Guild Wars Doppelganger [5], Renegade Shepard from Mass Effect 3 [6] and $S A-X$ in Metroid Fusion [42]. Yet, so far these have only been realized as crude approximations of the original player, as they mimic appearance, equipment, basic moves and/or skill sets by relying on heuristic, strategically rigid decisionmaking.

At the same time, machine learning approaches in video games that harness continuous improvement through simulated play have become popular, e.g. the deep reinforcement learning of Atari games [31], temporal difference learning of Backgammon [54] or the surpassing of human player performance in the board game that has been rated as not solvable by artificial intelligence methods for a long time; Go [48]. The field of player modeling has seen explorations of machine learning techniques for multiple purposes, prominently featuring prediction, classification or analysis [13, 14, 27, 53], to facilitate individual game interpretations, testing or for providing believable opponents. Still, the incorporation of machine learning approaches for generative player modeling for DDA and the resulting player experiences remain under-investigated. Holmgård et al. studied personas for player decision modeling $[19,20]$ that continually observe and adapt to human behavior in order to produce agents with different decisionmaking styles. These personas were realized via evolutionary linear perceptrons and compared to heuristic agents in a test bed 2D dungeon crawler game, resulting in a higher playerrated human-likeness. They also assessed player models when defined as "deviations from theoretically rational actions" in a study of Super Mario Bros. [1, 21] and clustered these by means of feature extraction. Using the same game, Ortega et al. [36] imitated human playing styles by means of Neuroevolution and Dynamic Scripting and reached higher scores of human-likeness than performance-directed AI agents, based on subjective judgments. Missura and Gärtner utilized Player Modeling in a 2D test bed shooter via Support Vector Machines acting as predictors for difficulty mismatches and enabling classical DDA parameter tuning based on the results [30]. In previous work, we were successful in showing that player model agents can yield significantly higher motivation compared with heuristic opponents in a short-term online study using the 2D platform fighter Korona:Nemesis [9, 39]. Based on these insights, player awareness about substituting individual players with DPBM agents in online multi-player matches was also assessed. In contrast to heuristic bots, DPBM agents turned out to be indistinguishable from their human precursors [37]. In addition, we contrasted different machine learning techniques in a player modeling study of the MMORPG Lineage $2[33,38]$. Deep learning offered the best individual prediction accuracy, facilitating the production of playing sessions that closely resemble the original behavior, as well as for differentiating between players. Consequently, we discussed the broader implications for the application of DPBM in: DDA (offering adaptation beyond parameter tuning; training players by exposing them to own strengths and weaknesses), player substitution (bridging online match disruption due to dropouts; providing more individually representative agents), automated game testing (enhancing the estimation of balancing issues by incorporating realistic human player behavior) and cheating detection (revealing behavior that is more likely to stem from undesirable third-party bots rather than players; yield- 
ing objective evidence based on behavior in cases of identity theft).

To the best of our knowledge, there is no prior work assessing the experience of players who continuously challenge themselves, where generative player modeling facilitates proficiency progress.

\section{APPROACH}

In contrast to the aforementioned studies, the approach presented in this work provides a medium- to long-term situated evaluation. We compare the deployment of player modeling through DPBM with traditional HPT and assess feasibility, approval and motivation in a complex AAA game through a highly ecologically valid field study. To facilitate realistic and generalizable results that avoid artificial laboratory study setting biases [44], we aimed for the deployment of our approach in a real-world setting in a fully fledged game with an existing community of players. In the following, we explain the construction of the recorded training data format, how it was informed by expert interviews, the DPBM architecture, and the study environment.

\section{Expert Interview}

In order to gain a more elaborate understanding of viable strategies, decision making and what behavior might lead to different play styles in Aion, one of the authors consulted 3 expert players of the game (each with 7-8 years of prior experience) and extracted the most important aspects qualitatively, using brief 1 hour semi-structured interviews over the course of one day. Apart from a less-structured introduction and follow-up discussion after each item, we asked the following questions:

- In a one-on-one situation against a (computer controlled opponent / human player), based on which factors do you decide which skill to use?

- How do you react when you are not able to execute your strategy?

- How would you approach an opponent of the same class that is equally proficient as yourself?

We analyzed the interview using an outcome-oriented structuring content analysis after Mayring [28] and consolidated the most expressive statements about factors that qualify as good indicators for decision making. The most descriptive factors as indicated by the experts are adherence to skill rotations and situational responsive decisions. Within rotations, expert players predominantly apply a specific set of preferred sequences of actions, e.g. ramping up damage by combinations of enhancing and weakening skills or controlling the opponent by a succession of restricting skills. Due to the large amount of possible skills or items to use (cf. Figure 2), these rotations can include complex chains of consecutive skills and/or contain sub-rotations. Responsive decisions denote the reaction to certain states that the player character or an opponent is in, e.g. healing oneself when hit points are low, removing restricting conditions on the character, increasing or reducing distance between characters or exploiting temporary conditions the opponent is in. They can also trigger more complex situation-specific rotations. The description of playstyle aspects by means of rotations and responsive decisions is not limited to this specific game, but broadly generalizes to the genre of action RPG games. We defined the DPBM state-action architecture on the basis of these factors, including player and target state information as crucial indicators for responsive decisions and previous skill information for positioning within rotations.

In combination, these factors compose the game state that is fed into the DPBM input layer, while the output layer is trained according to the respective skill that the player used in this situation (cf. Figure 3).

\section{Adaptive Instance Dungeon}

Instance dungeons are a major part of MMORPGs, as they can be entered numerous times, solo or in a group, to acquire experience points, equipment, currencies and/or other desirable items. As such, they provide a fertile testing ground for evaluating long-term motivation [52], since most often repeated or even continuous entries are required to reach higher-level goals. To gather expressive evidence of the motivational potential of DPBM, we developed the single-player adaptive instance dungeon Eternal Challenge that incorporates both traditional DDA aspects via HPT as well as DPBM. Within the instance, the players encountered various opponents that were clearly distinguishable by their visual appearance (cf Figure 1) and were adjusted through distinct parameters tuned by HPT (cf. Table 1). The underlying proficiency variable $\lambda$ approximated the player's performance level by being increased whenever he successfully completed Eternal Challenge and decreased at the characters death or temporal expiry of the countdown. This way, HPT produces a typical "rubberbanding" effect between player-specific thresholds of lack of challenge and excessive challenge, which is one of the most common ways to enable flow-states to arise in traditional DDA [47] and was constructed by following the inspiration of these approaches $[17,23,30,47]$ in combination with fine-tuning by the developers operating the server to find a range covering too easy, too hard and enough configurability in between for every observed player.

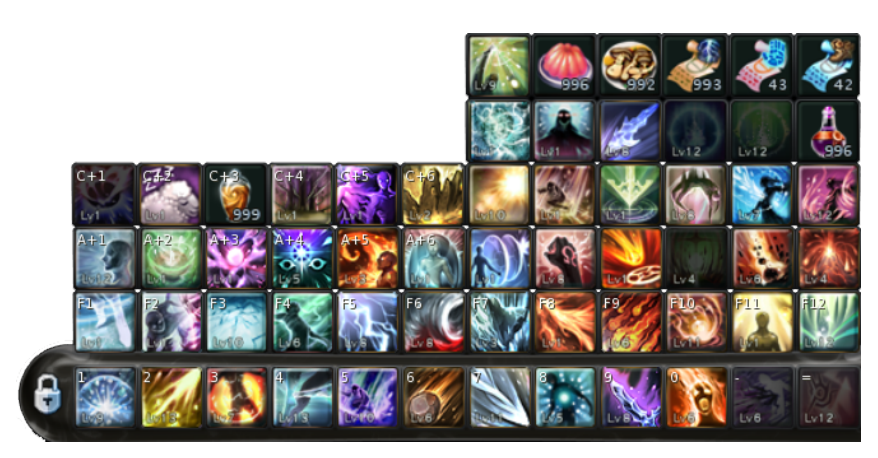

Figure 2. Exemplary arrangement of a subset of skills available to the Sorcerer class in Aion. Additionally, context-dependent skills (when the player or a target opponent is in a particular condition) and a multitude of items can be activated. 
Increased whenever Eternal Challenge

$\lambda$ difficulty level was completed successfully, decreased upon death or timeout.

With increased $\lambda$, the temporal

$\alpha \quad$ frequency

spawn delay of $\alpha$ opponents was decreased, resulting in an exponential increase of difficulty.

$\beta$ perseverance

With increased $\lambda$, hit points (HP) of $\beta$ opponents increased, making them harder / more time-consuming to defeat.

With increased $\lambda, \gamma$ opponents used $\gamma$ disturbance more actions that weaken the player, which decreases survivability, damage performance and increases the tension.

\section{DPBM}

No explicit parameter tuning was used, since network proficiency approximates the player's skill implicitly.

Table 1. DDA mechanisms of Eternal Challenge, mapping $\lambda$ to the difficulty of $\alpha, \beta, \gamma$, while DPBM seeks to emulate the player's behavior.

To avoid incentivizing players to perform badly on purpose, rewards (in the form of experience gained and the level-range of items dropped) were adjusted to be proportional to the difficulty level $\lambda$. Upon entering the instance, a 15-minute countdown started that expelled the player if it was not finished after expiration. Within this time limit, the player was expected to destroy a sturdy, non-responsive opponent $(\beta)$ that spawned additional, hostile enemies over time $(\alpha, \beta, \gamma)$ which had to be endured or defeated as well. As soon as the main opponent was defeated, an additional foe that utilizes DPBM appeared in an adjacent room. If the player managed to beat this opponent as well, rewards were distributed and the internal $\lambda$ level was raised accordingly. $\lambda$ had no theoretical, but a practical upper limit, since the game inherently restricts reaching damage per second values beyond a certain threshold.

\section{Deep Player Behavior Modeling}

When entering Eternal challenge, the recorded behavioral data from all preceding runs of the player was retrieved from the underlying database and fed into a feed-forward multilayer perceptron with backpropagation and a logistic sigmoid activation function (cf. Figure 3), where input and output layer size varied depending on the player's class, skill set and usage $(M=98.2, S D=15.1)$ input, $5 \times 10$ hidden, ( $M=76.2, S D=15.1)$ output nodes). The network was initialized randomly and trained over 1000 epochs, based on the insights of previous work $[38,39]$ and benchmarks prior to the study that indicated diminishing returns when further increasing the range of parameters. When encountering the DPBM opponent, the trained model was applied generatively to retrieve a set of action probabilities given the occurring state description at real-time. After a weighted choice, the resulting skill was executed, followed by querying the DPBM for the next situation, effectively approximating the learned behavior from the player's preceding battles. As movement was controlled heuristically, temporal-dynamics of behavior are not explicitly modeled, but behavior over time is modeled by focusing the sequencing of skill rotations and responsive decisions in each occurring state. In terms of the player modeling taxonomy of Yannakakis et al. [58], this implementation realizes a model-free (bottom-up) player modeling approach mapping gameplay data to actions via preference learning and classification. According to the player modeling description framework of Smith et al. [49], DPBM directly utilizes game actions (domain) to generate (purpose) individually (scope) modeled behavior by means of induced (source) training of machine learning techniques.

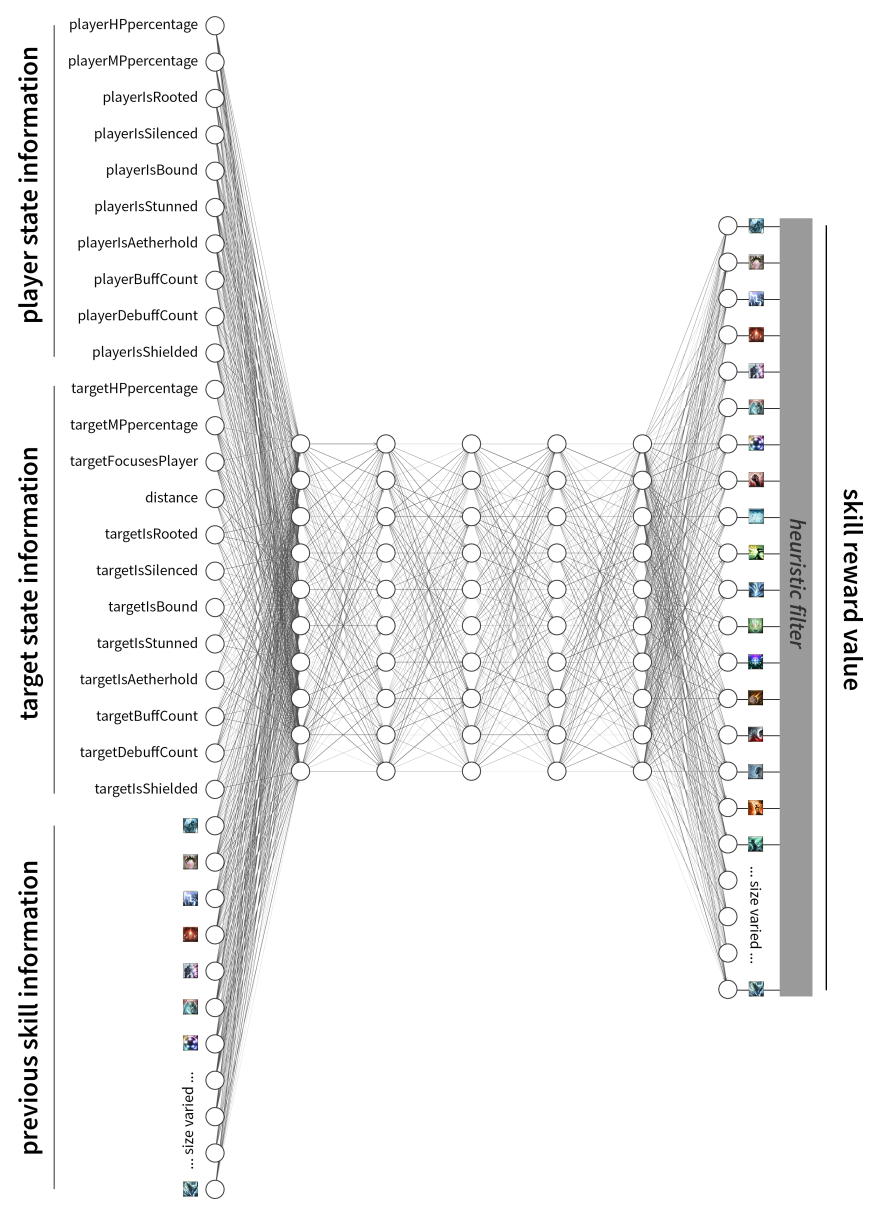

Figure 3. The DPBM architecture mapping game state (information about player, opponent and skill history) to action (skill usage) probabilities. The size of the input and output layers varied depending on the player's class, skill set and usage. The resulting action probability array is filtered heuristically by removing skills that are impossible to execute due to cool-down, MP shortage or other insufficient conditions. 


\section{STUDY}

In order to evaluate the DDA and intrinsic motivation capabilities of DPBM, we conducted an online field study following a within-subjects design over the course of four weeks. The adaptive instance was published on a private Aion game server. To ensure that the measured motivation is attributable to the DPBM approach, we took several precautions. In order to minimize novelty or anticipation bias, we did not announce the existence or concept of the instance prior release and chose a long-term study design. In addition, rewards constitute one of the biggest extrinsic motivators for long-term commitments [56] and thus a potentially high confounding factor when assessing intrinsic motivation. Therefore, the rewards of Eternal Challenge were kept conservative and approximated the amount of rewards in other available instances, i.e. players were not able to obtain something that they would not be able to elsewhere and did not acquire a higher reward-to-time-ratio. Findings of Deci et al. [12] also suggest that excessive extrinsic rewards can inhibit intrinsic motivation, the core factor of engagement and enjoyment [45, 41]. Finally, as interplay among players is a major motivating factor of MMORPGs [56], we excluded multi-player situations, leaderboards, high score lists or the publication of ranks and completion times during the study period to avoid complex potential biases in this early situated study. Although MMORPGs are designed to be about multi-player scenarios, occasions of playing solo occur on a regular basis and novel challenge paradigms should arguably be tested in basic, controllable setups before being extended to include additional factors, such as team play or competition.

\section{Measures}

For every single Eternal Challenge run performed by a player, we recorded state-action data for DPBM training (cf. Figure 3), instance completion times and results (failed or succeeded), as well as the training times and prediction accuracies of the DPBM. In addition, we logged entry counts and timestamps for all available instance dungeons for further activity comparisons. After the study period, a post-study questionnaire asked for player-reported assessments of perceived competence, interest-enjoyment, tension-pressure and effort-importance, following the Intrinsic Motivation Inventory (IMI) [29], for comparison between the traditional DDA parameters $\alpha, \beta, \gamma$, and the DPBM opponent. Each iteration of the questionnaire was explicitly headed by a display of the appearance of the corresponding opponent in order to assure correctly targeted responses (cf. Figure 1).

Additionally, the survey contained qualitative queries about the appreciation of - and strategies used against - the opponents, the impression of DPBM opponent's behavior in the players' own words, and a free field for additional remarks.

\section{Procedure}

The instance dungeon Eternal Challenge was introduced and released as part of a regular update to the private server. From then on, players of the community had the opportunity to enter it up to once daily, independent from entering different or additional instances. After four weeks, the recording of in-game data stopped and the post-study questionnaire was advertised on a message board associated with the server.

\section{Participants}

During the study period, $(n=171)$ participants entered Eternal Challenge resulting in 776 total instance runs. 30 players (17 men, 13 women (self-identified)) completed the optional post-study questionnaire.

\section{RESULTS}

Using a one-way RM ANOVA, we found significant effects for the IMI scores perceived competence, interest-enjoyment, tension-pressure and effort-importance between conditions $\alpha, \beta, \gamma$ and DPBM (cf. Table 2).

These outcomes were further evaluated using two-tailed paired t-tests (cf. Table 3, Figure 4). Employing conservative Bonferroni correction, $p$-values were multiplied with the amount of repeated comparisons. DPBM received significantly higher scores of interest-enjoyment and effort-importance compared to all HPT opponents, resulting in mostly large effect sizes after Cohen. It also outperformed $\alpha$ and $\beta$ significantly in terms of tension-pressure with medium effect sizes.

$$
\begin{array}{rllc}
\text { perceived competence } & F(3,26)=3.59 & p<.05 & \eta^{2}=0.29 \\
\text { interest-enjoyment } & F(3,26)=8.75 & p<.01 & \eta^{2}=0.5 \\
\text { tension-pressure } & F(3,26)=3.37 & p<.05 & \eta^{2}=0.28 \\
\text { effort-importance } & F(3,26)=5.63 & p<.01 & \eta^{2}=0.39
\end{array}
$$

Table 2. ANOVA results ( $F(d f 1, d f 2)-, p-$ values, $\eta^{2}$ for effect size) of the Intrinsic Motivation Inventory between the different opponents.

On average, players spent $(M=7.71, S D=2.49)$ minutes in the instance and used up to $91(M=21.1, S D=11.6)$ different skills against the DPBM opponent. Model training times lasted $(M=2018, S D=3692) \mathrm{ms}$ per session with $(M=8.75, S D=$ 3.34) $\mathrm{ms}$ per recorded skill.

To assess the objective quality of the underlying machine learning model and render it comparable to related approaches, $80 \%$ of the data recorded until any given time of entry into the instance was used for training, whereas $20 \%$ served for a routine initial test, resulting in $(M=60.64, S D=22.57) \%$ prediction accuracy. 

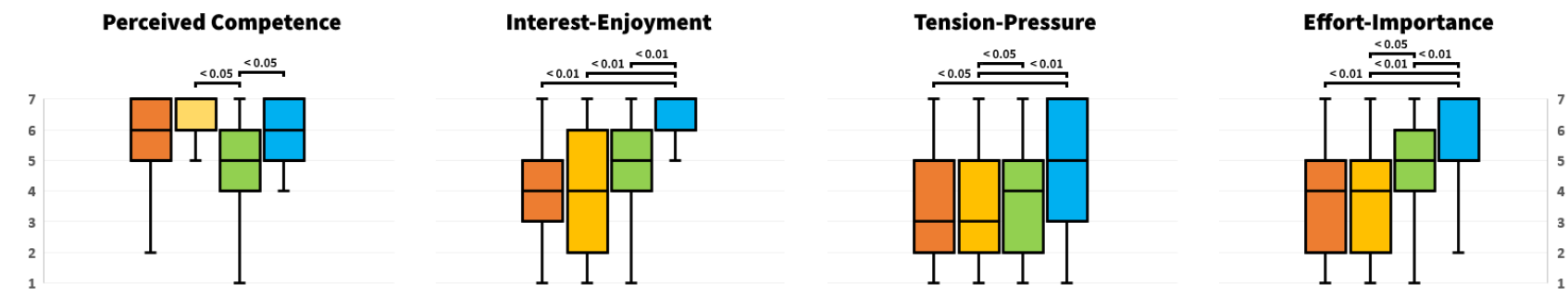

$\alpha$ : frequency

$\beta$ : perseverance

ү: disturbance

б:DPBM

Figure 4. Intrinsic motivation inventory (IMI) results for the compared DDA variables. Includes medians (center marks), standard deviations (boxes), minimal and maximal values (whiskers) and significant difference markers.

Compared to all 35 available instances in the game, Eternal Challenge (EC) became the most popular instance by daily numbers of players over the duration of the study (cf. Figure 5), as chi-square goodness of fit tests show (cf. Table 4), assuming equal proportions. Even when omitting the first quarter to counteract a presumable novelty bias in the distinct initial spike, EC still outmatched all alternatives.

\begin{tabular}{|c|c|c|c|}
\hline $\begin{array}{r}\text { interest- } \\
\text { enjoyment }\end{array}$ & $\begin{array}{l}D P B M \\
(M=6.17, \\
S D=1.11)\end{array}$ & $\begin{array}{r}\text { effort- } \\
\text { importance }\end{array}$ & $\begin{array}{l}D P B M \\
(M=5.87, \\
S D=1.63)\end{array}$ \\
\hline $\begin{array}{c}\alpha \\
(M=4, \\
S D=1.51)\end{array}$ & $\begin{array}{l}p=.000 \\
d=1.64\end{array}$ & $\begin{array}{c}\alpha \\
(M=4.04, \\
S D=2.03)\end{array}$ & $\begin{array}{c}p=.006 \\
d=.99\end{array}$ \\
\hline $\begin{array}{c}\beta \\
(M=4.04, \\
S D=2.06)\end{array}$ & $\begin{array}{l}p=.001 \\
d=1.23\end{array}$ & $\begin{array}{c}\beta \\
(M=3.91, \\
S D=1.83)\end{array}$ & $\begin{array}{l}p=.000 \\
d=1.13\end{array}$ \\
\hline $\begin{array}{c}\gamma \\
(M=4.78, \\
S D=1.76)\end{array}$ & $\begin{array}{l}p=.01 \\
d=.95\end{array}$ & $\begin{array}{c}\gamma \\
(M=4.91, \\
S D=1.81)\end{array}$ & $\begin{array}{c}p=.004 \\
d=.56\end{array}$ \\
\hline $\begin{array}{l}\text { tension- } \\
\text { pressure }\end{array}$ & $\begin{array}{l}D P B M \\
(M=4.91, \\
S D=2.15)\end{array}$ & $\begin{array}{r}\text { perceived } \\
\text { competence }\end{array}$ & $\begin{array}{l}D P B M \\
(M=5.83, \\
S D=1.07)\end{array}$ \\
\hline $\begin{array}{c}\alpha \\
(M=3.39, \\
S D=1.8)\end{array}$ & $\begin{array}{c}p=.049 \\
d=.77\end{array}$ & $\begin{array}{c}\alpha \\
(M=5.65, \\
S D=1.67)\end{array}$ & $p>.05$ \\
\hline $\begin{array}{c}\beta \\
(M=3.39, \\
S D=1.8)\end{array}$ & $\begin{array}{c}p=.007 \\
d=.77\end{array}$ & $\begin{array}{c}\beta \\
(M=5.91, \\
S D=1.35)\end{array}$ & $p>.05$ \\
\hline $\begin{array}{c}\gamma \\
(M=4.17, \\
S D=1.85)\end{array}$ & $p>.05$ & $\begin{array}{c}\gamma \\
(M=4.65, \\
S D=1.72)\end{array}$ & $p>.05$ \\
\hline
\end{tabular}

Table 3. Means, standard deviations and significant t-test results after Bonferroni correction ( $p$-values and Cohen's $d$ for effect size, $d f=29$ ) of the Intrinsic Motivation Inventory between the different opponents.
For the qualitative remarks, we used a structuring content analysis after Mayring [28] to assess the effect of challenging the DPBM opponent. Players were asked to state their general impression and opinion freely, without confounding or influencing questions. From the utilizable statements, 31.8\% describe an appropriate challenge (e.g. "quite easy at first but afterwards I really was busy thinking about how I approach him", "it's almost as good as I am"), while 9.1\% depict it as slightly too high or slightly to low. $13.6 \%$ emphasize a notable entertaining factor, whereas $4.4 \%$ declare that this encounter did not appeal to them. Although the behavior or decision-making of the DPBM opponent was never explicitly stated or explained during the study, $36.4 \%$ of players ascribed the ability to learn from previous battles and the adaptation to the player's own behavior, combos, rotations and/or strategies to their enemy (e.g. "at first he randomly used skills that I also used, later he added my combos", "tried to replicate my own skills and techniques", "it was hilarious when I played against myself").

$$
\text { During complete study period: }
$$

\begin{tabular}{l|l|l}
$E C$ vs. \#2 & $\chi^{2}(1, n=1007)=58.64$ & $p<0.01$ \\
$E C$ vs. \#3 & $\chi^{2}(1, n=902)=134.26$ & $p<0.01$ \\
EC vs. \#4 & $\chi^{2}(1, n=856)=181.35$ & $p<0.01$
\end{tabular}

After first quarter of study period:

\begin{tabular}{l|c|c} 
EC vs. \#2 & $\chi^{2}(1, n=627)=4.48$ & $p=0.03$ \\
EC vs. \#3 & $\chi^{2}(1, n=526)=45.09$ & $p<0.01$ \\
EC vs. \#4 & $\chi^{2}(1, n=493)=70.93$ & $p<0.01$
\end{tabular}

Table 4. Chi-square goodness of fit tests between Eternal Challenge and the second, third and fourth most popular instance. Less popular instances have shown similarly significant results, but have been omitted for the sake of readability.

\section{DISCUSSION}

Our results indicate distinct effects on approval and intrinsic motivation for DPBM opponents, as well as effects on longterm commitment for the presence of DDA in general. We were successful in evidencing significantly higher motivation for players to enter adaptive instance dungeons compared to static alternatives over considerable duration of successive play sessions and report indications that DPBM attributes significantly more to this preference than traditional DDA parameter tuning. 


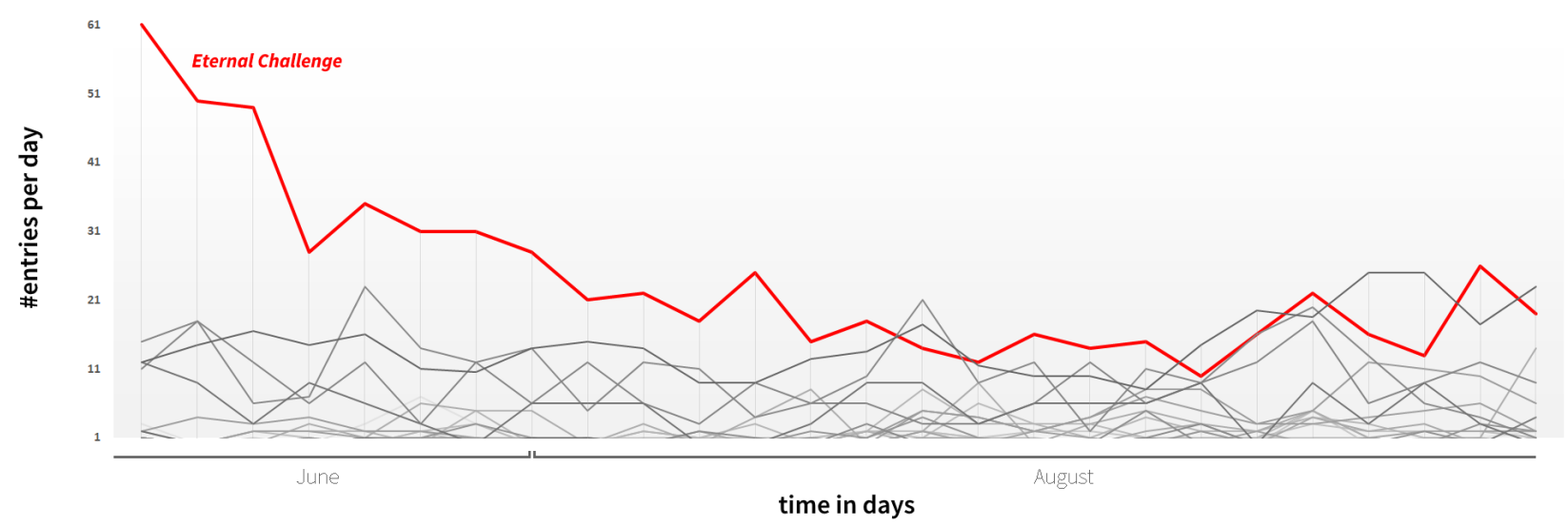

Figure 5. Daily number of unique players entering Eternal Challenge compared to all other available instances during the study period.

This insight is based on notably high absolute IMI scores and significant differences compared to conventional DDA techniques. The outcome that DPBM outperformed HPT in terms of interest-enjoyment indicates a high "fun factor", while tension-pressure and effort-importance highlight the considerable challenge, leading to an overall higher intrinsic motivation and linked potential to induce flow. The actual implicit DDA capabilites of DPBM are backed by qualitative statements that reveal an appropriate challenge, a noticeable difficulty adjustment over time and the perception of playing against an equal opponent that facilitates rethinking of habitual behavior. This work also demonstrates the technical applicability of largescale, long-term generative player modeling with reasonable training times and accuracies.

Overall, our work provides quantitative and qualitative empirical evidence supporting our initial hypotheses about facilitating long-term motivation potential, capabilities for enabling DDA and individual representation, indicating the following responses to the respective research questions:

- We measured a substantially and consistent motivation achieved with the support of DPBM over the mediumto long-term.

- The measured intrinsic motivation of challenging a DPBM-fuelled opponent significantly exceeded traditional HPT.

- Players perceive behavior from DPBM as representative of - or comparable to - their own decision-making.

\section{Limitations and Future Work}

The mixed-bag fashion of the instance, which resulted from aiming to maintain an ecologically-valid realistic instance design, results in a combined experience of HPT and DPBM opponents that might influence the participants' assertions. This study design was selected due to the long-term period of the study in a community where players know each other, rendering a between-subjects design confounding, since players would have exchanged views about the different conditions and/or complained about unjust treatments.
To further corroborate evidence and to gain a clear comparison between the different HPT factors and DPBM, the experiment should be replicated to manifest a control group (mutually exclusive from this player base) in which no DPBM (or HPT) is present. Apart from that, the Intrinsic Motivation Inventory was designed to measure single sessions within experiments. While it was not explicitly developed for this study's setup, we found it to be the most appropriate questionnaire to assess motivation, as there is no validated reflective long-term motivation questionnaire that does not have to be raised after every single session (which was omitted in favor of ecological validity).

Based on our achievements and outcomes, we are looking forward to extending the scope of using DPBM in video games to enabling personalized, adaptive challenges that go beyond oneon-one situations to encompass interactions between different players and consider both competitive as well as cooperative interplay. DPBM agents could be deployed in multi-player scenarios where groups are challenged to deal with effects between player modeled opponents or utilized to support teamfights between human players, as equivalent reinforcements. Additionally, we plan to construct a one-dimensional proficiency metric that maps DPBM configurations to estimated competence in a game, in order to offer players more unique DDA encounters stemming from different players with similar proficiency. Using the considerably large data set recorded in this study, we seek to benchmark several alternative machine learning techniques as core mechanisms for the underlying player modeling (e.g. recurrent, deep belief, GAN or contextdriven LSTM networks), to be able to give practical statements about applicability concerning temporal requirements and resulting accuracy. Furthermore, we envision DPBM as an effective instrument for elevating autonomous game testing and balancing, since actual, precise player behavior can be simulated, and temporary substitutions or continuations of disconnected players in online matches can be facilitated to minimize game experience disruptions. 


\section{CONCLUSION}

We presented the design and implementation of an adaptive instance dungeon in the MMORPG Aion to evaluate a novel, implicit take on Dynamic Difficulty Adjustment that is not dependent on manually composed parameter tuning, but affords a continually adapting challenge through Deep Player Behavior Modeling. In an extensive medium- to long-term study ( $n=171$ over the course of four weeks) we contrasted an opponent applying DPBM to traditional DDA parameter tuning and can report significantly higher intrinsic motivation stemming from the unique game experience of being confronted with strategic behaviors that mirror one's own patterns. Qualitative statements reinforce the approval and positive experience of DPBM, while the consistent and dominant usage of the instance throughout the whole study period reflects its potential to elevate long-term motivation and commitment. Regarding the technical applicability of the approach, we report on the DPBM architecture, its accuracy and data structure and give an estimation about the temporal demand, yielding real-time potential.

According to the guidelines of transparent statistics, the collected data of this approach, as well as its implementation, will be made openly available upon publication, using an openaccess repository.

\section{ACKNOWLEDGMENTS}

We would like to thank all participants, testers, Beyond Aion for hosting the instance dungeon and Philipp Krüger for his collaboration, advice and porting. This work was funded by the German Research Foundation (DFG) as part of Collaborative Research Center (SFB) 1320 EASE - Everyday Activity Science and Engineering, University of Bremen (http://www.easecrc.org/), subproject H2.

\section{REFERENCES}

[1] Nintendo Research Development 4. 1985. Super Mario Bros. Game [NES]. (13 September 1985). Nintendo, Kyōto, Japan.

[2] Ernest Adams. 2002. Balancing Games with Positive Feedback. Gamasutra. com, January 4 (2002).

[3] Dennis Ang and Alex Mitchell. 2017. Comparing Effects of Dynamic Difficulty Adjustment Systems on Video Game Experience. In Proceedings of the Annual Symposium on Computer-Human Interaction in Play. ACM, 317-327.

[4] Dennis Ang and Alex Mitchell. 2019. Representation and Frequency of Player Choice in Player-Oriented Dynamic Difficulty Adjustment Systems. In Proceedings of the Annual Symposium on Computer-Human Interaction in Play. ACM, 589-600.

[5] ArenaNet. 2005. Guild Wars. Game [PC]. (28 April 2005). ArenaNet, Bellevue, WA. Played 2019.

[6] BioWare. 2012. Mass Effect 3. Game [PC,XBox360,PS3,WiiU]. (6 March 2012). BioWare, Edmonton, Kanada.
[7] Capcom Production Studio 4. 2005. Resident Evil 4. Game [Gamecube]. (11 January 2005).

[8] Thomas Constant and Guillaume Levieux. 2019. Dynamic Difficulty Adjustment Impact on Players' Confidence. In Proceedings of the 2019 CHI Conference on Human Factors in Computing Systems. ACM, 463.

[9] Nevermind Creations. 2019. Korona:Nemesis. Game [PC]. (18 August 2019).

[10] Mihaly Csikszentmihalyi. 2013. Flow: The psychology of happiness. Random House.

[11] Thomas Debeauvais. 2016. Challenge and retention in games. Ph.D. Dissertation. UC Irvine.

[12] Edward L Deci, Richard Koestner, and Richard M Ryan. 1999. A meta-analytic review of experiments examining the effects of extrinsic rewards on intrinsic motivation. Psychological bulletin 125, 6 (1999), 627.

[13] Anders Drachen, Alessandro Canossa, and Georgios N Yannakakis. 2009. Player modeling using self-organization in Tomb Raider: Underworld. In 2009 IEEE symposium on computational intelligence and games. IEEE, 1-8.

[14] Anders Drachen, Rafet Sifa, Christian Bauckhage, and Christian Thurau. 2012. Guns, swords and data: Clustering of player behavior in computer games in the wild. In 2012 IEEE conference on Computational Intelligence and Games (CIG). IEEE, 163-170.

[15] Nintendo EAD. 1987. Zelda II: The Adventure of Link. Game [NES]. (14 January 1987). Nintendo EAD, Kyoto, Japan.

[16] Nintendo EAD. 2014. Mario Kart 8. Game [WiiU,Switch]. (29 May 2014). Nintendo EAD, Kyoto, Japan. Played 2019.

[17] William Rao Fernandes and Guillaume Levieux. 2019. $\delta$-logit: Dynamic Difficulty Adjustment Using Few Data Points. In Joint International Conference on Entertainment Computing and Serious Games. Springer, 158-171.

[18] Julian Frommel, Fabian Fischbach, Katja Rogers, and Michael Weber. 2018. Emotion-based Dynamic Difficulty Adjustment Using Parameterized Difficulty and Self-Reports of Emotion. In Proceedings of the 2018 Annual Symposium on Computer-Human Interaction in Play. ACM, 163-171.

[19] Christoffer Holmgård, Antonios Liapis, Julian Togelius, and Georgios N Yannakakis. 2014a. Evolving personas for player decision modeling. In 2014 IEEE Conference on Computational Intelligence and Games. IEEE, 1-8.

[20] Christoffer Holmgård, Antonios Liapis, Julian Togelius, and Georgios N Yannakakis. 2014b. Generative agents for player decision modeling in games.. In FDG. Citeseer. 
[21] Christoffer Holmgård, Julian Togelius, and Georgios N Yannakakis. 2013. Decision making styles as deviation from rational action: A super mario case study. In Ninth Artificial Intelligence and Interactive Digital Entertainment Conference.

[22] Dayana Hristova. 2017. Dynamic difficulty adjustment (DDA) in first person shooter (FPS) games. (2017).

[23] Robin Hunicke. 2005. The case for dynamic difficulty adjustment in games. In Proceedings of the 2005 ACM SIGCHI International Conference on Advances in computer entertainment technology. ACM, 429-433.

[24] Robin Hunicke and Vernell Chapman. 2004. AI for Dynamic Difficulty Adjustment in Games.

[25] Changchun Liu, Pramila Agrawal, Nilanjan Sarkar, and Shuo Chen. 2009. Dynamic difficulty adjustment in computer games through real-time anxiety-based affective feedback. Int. Jrnl. of Human-Computer Interaction 25, 6 (2009), 506-529.

[26] Ricardo Lopes, Ken Hilf, Luke Jayapalan, and Rafael Bidarra. 2013. Mobile adaptive procedural content generation. In Proceedings of the fourth workshop on Procedural Content Generation in Games (PCG 2013), Chania, Crete, Greece.

[27] Tobias Mahlmann, Anders Drachen, Julian Togelius, Alessandro Canossa, and Georgios N Yannakakis. 2010. Predicting player behavior in tomb raider: Underworld. In Proceedings of the 2010 IEEE Conference on Computational Intelligence and Games. IEEE, 178-185.

[28] Philipp Mayring. 2010. Qualitative inhaltsanalyse. In Handbuch qualitative Forschung in der Psychologie. Springer, 601-613.

[29] Edward McAuley, Terry Duncan, and Vance V Tammen. 1989. Psychometric properties of the Intrinsic Motivation Inventory in a competitive sport setting: A confirmatory factor analysis. Research quarterly for exercise and sport 60, 1 (1989), 48-58.

[30] Olana Missura and Thomas Gärtner. 2009. Player Modeling for Intelligent Difficulty Adjustment. In Discovery Science, João Gama, Vítor Santos Costa, Alípio Mário Jorge, and Pavel B. Brazdil (Eds.). Springer Berlin Heidelberg, Berlin, Heidelberg, 197-211.

[31] Volodymyr Mnih, Koray Kavukcuoglu, David Silver, Andrei A Rusu, Joel Veness, Marc G Bellemare, Alex Graves, Martin Riedmiller, Andreas K Fidjeland, Georg Ostrovski, and others. 2015. Human-level control through deep reinforcement learning. Nature 518, 7540 (2015), 529.

[32] Fausto Mourato, Fernando Birra, and Manuel Próspero dos Santos. 2014. Difficulty in action based challenges: success prediction, players' strategies and profiling. In Proceedings of the 11th Conference on Advances in Computer Entertainment Technology. ACM, 9.

[33] NCsoft. 2003. Lineage 2. Game [PC]. (1 October 2003). NCSoft, Seongnam, South Korea.
[34] NCsoft. 2008. Aion. Game [PC]. (25 September 2008). NCSoft, Seongnam, South Korea. Played August 2019.

[35] Pedro A Nogueira, Vasco Torres, Rui Rodrigues, Eugénio Oliveira, and Lennart E Nacke. 2016. Vanishing scares: biofeedback modulation of affective player experiences in a procedural horror game. Journal on Multimodal User Interfaces 10, 1 (2016), 31-62.

[36] Juan Ortega, Noor Shaker, Julian Togelius, and Georgios N Yannakakis. 2013. Imitating human playing styles in super mario bros. Entertainment Computing 4, 2 (2013), 93-104

[37] Johannes Pfau, Jan David Smeddinck, Ioannis Bikas, and Rainer Malaka. 2020. Bot or not? User Perceptions of Player Substitution with Deep Player Behavior Models. In Proceedings of the 2020 CHI Conference on Human Factors in Computing Systems. ACM.

[38] Johannes Pfau, Jan David Smeddinck, and Rainer Malaka. 2018. Towards Deep Player Behavior Models in MMORPGs. In Annual Symp. on Computer-Human Interaction in Play Ext. Abstracts (CHI PLAY'18). ACM, New York, NY, USA, 381-92.

[39] Johannes Pfau, Jan David Smeddinck, and Rainer Malaka. 2019. Deep Player Behavior Models: Evaluating a Novel Take on Dynamic Difficulty Adjustment. In Extended Abstracts of the 2019 CHI Conference on Human Factors in Computing Systems. ACM, LBW0171.

[40] Mike Preuss, Thomas Pfeiffer, Vanessa Volz, and Nicolas Pflanzl. 2018. Integrated Balancing of an RTS Game: Case Study and Toolbox Refinement. In 2018 IEEE Conference on Computational Intelligence and Games (CIG). IEEE, 1-8.

[41] Andrew K Przybylski, C Scott Rigby, and Richard M Ryan. 2010. A motivational model of video game engagement. Review of general psychology 14, 2 (2010), 154-166.

[42] Nintendo RD1. 2002. Metroid Fusion. Game [GBA]. (18 November 2002). Nintendo RD1, Kyoto, Japan.

[43] Andrew Rollings and Ernest Adams. 2003. Andrew Rollings and Ernest Adams on game design. New Riders.

[44] Robert Rosenthal and Kermit L Fode. 1963. The effect of experimenter bias on the performance of the albino rat. Behavioral Science 8, 3 (1963), 183-189.

[45] Richard M Ryan and Edward L Deci. 2000. Intrinsic and extrinsic motivations: Classic definitions and new directions. Contemporary educational psychology 25, 1 (2000), 54-67.

[46] Lingdao Sha, Souju He, Junping Wang, Jiajian Yang, Yuan Gao, Yidan Zhang, and Xinrui Yu. 2010. Creating appropriate challenge level game opponent by the use of dynamic difficulty adjustment. In 2010 Sixth International Conference on Natural Computation, Vol. 8. IEEE, 3897-3901. 
[47] Noor Shaker, Julian Togelius, and Georgios N Yannakakis. 2016. The experience-driven perspective. In Procedural Content Generation in Games. Springer, 181-194.

[48] David Silver, Aja Huang, Chris J. Maddison, Arthur Guez, and Laurent Sifre et al. 2016. Mastering the game of Go with deep neural networks and tree search. Nature 529 (2016), 484-489.

[49] Adam M. Smith, Chris Lewis, Kenneth Hullet, Gillian Smith, and Anne Sullivan. 2011. An Inclusive View of Player Modeling. In Proceedings of the 6th International Conference on Foundations of Digital Games (FDG '11). ACM, New York, NY, USA, 301-303.

[50] David Stammer, Tobias Günther, and Mike Preuss. 2015. Player-adaptive spelunky level generation. In 2015 IEEE Conference on Computational Intelligence and Games (CIG). IEEE, 130-137.

[51] Alexander Streicher and Jan D. Smeddinck. 2016. Personalized and Adaptive Serious Games. In Entertainment Computing and Serious Games, Ralf Dörner, Stefan Göbel, Michael Kickmeier-Rust, Maic Masuch, and Katharina Zweig (Eds.). Lecture Notes in Computer Science, Vol. 9970. Springer International Publishing, Cham, 332-377.

[52] Mirko Suznjevic and Maja Matijasevic. 2010. Why MMORPG players do what they do: relating motivations to action categories. International Journal of Advanced Media and Communication 4, 4 (2010), 405-424.

[53] Marco Tamassia, William Raffe, Rafet Sifa, Anders Drachen, Fabio Zambetta, and Michael Hitchens. 2016. Predicting player churn in destiny: A hidden markov models approach to predicting player departure in a major online game. In 2016 IEEE Conference on Computational Intelligence and Games (CIG). IEEE, $1-8$.

[54] Gerald Tesauro. 1994. TD-Gammon, a self-teaching backgammon program, achieves master-level play. Neural computation 6, 2 (1994), 215-219.

[55] Valve. 2008. Left 4 Dead. Game [PC]. (18 November 2008). Valve, Bellevue, WA, USA. Played 2017.

[56] Hao Wang and Chuen-Tsai Sun. 2011. Game reward systems: Gaming experiences and social meanings.. In DiGRA Conference, Vol. 114.

[57] Su Xue, Meng Wu, John Kolen, Navid Aghdaie, and Kazi A Zaman. 2017. Dynamic difficulty adjustment for maximized engagement in digital games. In Proceedings of the 26th International Conference on World Wide Web Companion. International World Wide Web Conferences Steering Committee, 465-471.

[58] Georgios N Yannakakis, Pieter Spronck, Daniele Loiacono, and Elisabeth André. 2013. Player modeling. Schloss Dagstuhl-Leibniz-Zentrum fuer Informatik. 